\title{
Performance Analysis of VoIP Codecs over BE WiMAX Network
}

\author{
M. Imran Tariq, M. Ajmal Azad, Razvan Beuran, and Yoichi Shinoda
}

\begin{abstract}
IEEE802.16 technology due to its outstanding larger coverage area, high data rates, inexpensive equipments, ease of deployment and guaranteed QoS make it a suitable candidate for future broadband wireless access networks. The Mobile WiMAX has been designed to provide Broadband Internet service to mobile users. The VoIP is flexible and provides low cost telephony to customers over the existing IP infrastructure. However, there are still many challenges that need to be addressed to provide a steady and good quality voice connection over the best-effort Internet. In this paper we evaluate the performance of different VoIP codecs over the best effort WiMAX network. The network performance metrics such as jitter, one way delay, and packet loss and user perception metric that is Mean Opinion Score have been used to evaluate the performance of VoIP codecs. The simulation is performed in QualNet simulator with varying values of Packet size, number of calls and jitter buffer sizes. Our results indicate that varying the jitter buffer size and packetization time affects the quality of voice over the best effort network.
\end{abstract}

Index Terms-WiMAX, VoIP, BE, MOS, QoS, RTP, QualNet.

\section{INTRODUCTION}

Multimedia applications are gaining much of the user attention with the advent of new broadband technologies [1]. In recent decades user desires have switched from net surfing and email to multimedia services, such as VoIP \& video conferencing and video streaming, etc. To address the specific user needs for rich multimedia applications, the service providers are looking for broadband wireless network. The IEEE 802.16 standard [2] [3] has been designed as an access network to fulfill the user needs of multimedia applications. WMAN provides cost effective infrastructure to service providers and promised QoS to end users without increasing the complexity in the core network as well as at the user side. WiMAX is easy to deploy and integrate with the existing IP core network, which acts as a backbone infrastructure. The IP core offers the support of advanced technologies and protocols to WiMAX that fulfills the required Quality of Service (QoS) and security features [4].

Recently, VoIP over WiMAX has been emerging as an

Manuscript received July 9, 2012; revised November 10, 2012. This work was supported by Japan Advanced Institute of Science and Technology, 1-1 Asahidai, Nomi, Ishikawa, 923-1292 JAPAN.

M. Imran Tariq and Yoichi Shinoda are with Japan Advanced Institute of Science and Technology, (JAIST) 1-1 Asahidai, Nomi, Ishikawa, 923-1292 Japan (e-mail: imran@jaist.ac.jp).

M. Ajmal Azad is with INESCPORTO, Faculty of Engineering, University of Porto, Portugal (e-mail: mazad@inescporto.pt).

Razvan Beuran is a researcher with National Institute of Information and Communication Technology (NICT), Ishikawa Japan. infrastructure to provide broadband wireless voice service with cost efficiency reliability and guaranteed quality of service. However, the supporting VoIP over WiMAX network faces major challenges as compared to its counterpart wire DSL network. Therefore the VoIP over WiMAX raises several deployment issues concerning the network architecture, system design, network capacity, configurations and QoS provisioning.

The purpose of this study is to evaluate the performance of VoIP codecs over the best effort scheduling class in WiMAX network, and examine the capability of a WiMAX network to deliver sufficient QoS to voice and data applications under different network conditions and load. The adopted methodologies include creating the WiMAX network, deploying the required applications for streaming video, FTP and VoIP telephony, the configuration of QoS parameters within the WiMAX core network as well as in the WiMAX access network, and QoS configurations within the WiMAX core network for voice applications. Simulations are performed using the QualNet simulator for different codecs under different packetization time, buffer size and with different network load.

The rest of the paper is organized as follows. In section II, we survey and comment on closely related work. In section III, we present our simulation design model; describe our evaluation methodology and some important configuration parameters for both the base station and the application. In section IV, we present our results and analyze the performance of a given VoIP application under different network conditions. Finally, section V concludes the paper, with outline of future work.

\section{RELATED WORK}

Many studies have been conducted in WiMAX to evaluate and analyze the VoIP performance, one closely related work to our, was published by Ricardo et al in [5], the authors measured the capacity of WiMAX link using BE and the performance of mixed traffic. In this study, the authors did not evaluate the VoIP performance regarding RTP jitter and delay. Fauzia et al, in [6], identified and defined a mechanism for free VoIP flows in a wireless network. Their main aim was to block these calls in entering network or to impose charges to obtain services. Another study by K.A. shuaib [4], identified the mobility influence over all throughput, the packet loss and delay with the main focus on signal strength. The researcher does not report the results with respect to the VoIP flows and QoS parameters. Scalabrino et al, [1], in their pioneer work, focused on VoIP performance using testbed. In this study, the authors mainly concentrated on the call quality measurement 
using the R-factor instead of the MOS. As I. Adhicandra stated in [7], with the use of ertPS instead of the UGS scheduling class, the BE performance can be increased because ertPS use a silence suppression mechanism. In this paper, the authors only consider the data over BE and also do not provide detail about the voice codec.

In this study, we explore the same methodology as in [5]. However, we evaluate the performance of various VoIP codecs with different performance metrics, and especially with and without RTP jitter buffer effects. Also, we evaluate the variant packet stay time in the jitter buffer, in order to select best one to experiments. Compared with the other works mentioned above, the measurements reports for the Mobile WiMAX are very rare. To the best of our knowledge, our study is one of the first which analyzes the performance of the VoIP codecs over the BE traffic class with respect to the RTP metrics.

\section{METHODLOGY}

In our study, we use the QualNet 4.5 simulator [8], which has a support of fixed and mobile WiMAX, to analyze the performance of VoIP under a given scenario. We designed two scenarios, one for the evaluation of the VoIP traffic behaviors over the BE class with the RTP jitter buffer, and the second one without it.

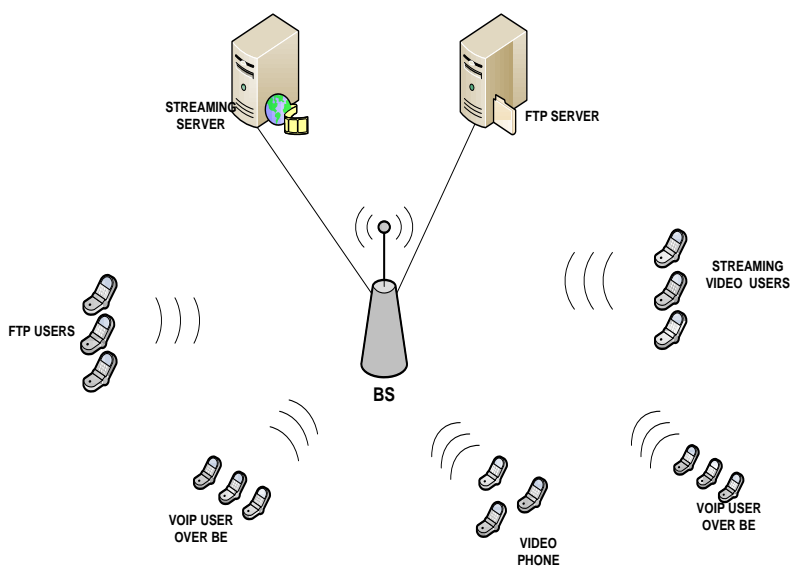

Fig. 1. Network topology for simulation experiment.

As shown in Fig. 1 and Table I, there is one BS, while the number of SSs varies up to 40. Others network configuration parameters are used in the simulation are defined in Table I. We assume that there are three groups of users as depicted in Fig. 1. They are placed around the BS in a circular fashion. The first group consist of 10 users who are obtaining a YouTube video stream from a video server (the Video traffic generator acts as a background video stream server) at 314 kbps $(320 * 240,24 \mathrm{f} / \mathrm{s})$ and some of them have smart phone video at $324 \mathrm{kbps}(320 * 240,24 \mathrm{f} / \mathrm{s})$, almost which both of them have the same data rate with a $20 \mathrm{~ms}$ mean inter-packet arrival time. The second group also contains 10 active users during the whole simulation time. Half of them are using the FTP server and having the file transfer service only in the downlink direction using TCP protocol at the rate of $512 \mathrm{kbps}$ and video traces of one clip at a $410 \mathrm{kpbs}$ average data rate and in peak condition less than $4 \mathrm{Mbps}$ because the leaky bucket is not used, is run on the rest of users within the same group.
TABLE I: NETWORK SIMULATION PARAMETERS

\begin{tabular}{ll} 
Description & Values \\
Terrain size & $1000 \times 1000 \mathrm{~m}$ \\
Number of cells & 1 \\
Number of base station & 1 \\
Number of SSs & 40 \\
Operating frequency & $2.4 \mathrm{GHz}$ \\
System bandwidth & $5 \mathrm{MHz}$ \\
Frame size (msec) & 5 \\
Frame size ratio of DL to UL & $2: 1$ \\
Phy Scheme & OFDMA \\
Duplex scheme & TDD \\
Modulation Technique & $64 \mathrm{QAM}, 16 \mathrm{QAM}, \mathrm{BPSK} / \mathrm{QPSK}$ \\
BS transmit power & $43 \mathrm{dBm}$ \\
SS transmit power & $20 \mathrm{dBm}$ \\
Simulation time & $1000 \mathrm{~s}$ \\
\hline
\end{tabular}

The last and important group consists of 20 users, and has VoIP flows (the term flows interchangeably to nodes) with different date rates depending on the codecs selection and requirements. These users are using the Google talk VoIP application for communication; in simple word we are taking Google talk as a reference VoIP application to evaluate the performance under WiMAX networks. All these VoIP flows are running over the best effort scheduling class, which has lowest priority among all scheduling classes, because the Google talk is freely available due to this much popular in the broadband users. The referenced application uses different types of audio codecs such as (G711, PCMA, PCMU, iLBC, G729, G723 etc.), for further study referred to [9], to provide good voice quality to users, and codec selection depending on the available bandwidth. To evaluate the VoIP codecs performance in the WiMAX network, we are taking G711, G726, G728, G729 and G723 with data rates of 64kbps, $32 \mathrm{Kbps}$ or $24 \mathrm{kbps}, 16 \mathrm{kbps}, 8 \mathrm{kbps}$ and $5.3 \mathrm{kbps}$ respectively. For those codecs, standard voice frames duration is used during the whole simulation time.

\section{RESULT AND DISCUSSION}

We evaluate the performance of the VoIP codecs in WiMAX using QualNet simulator. In this section, we discuss the performance of VoIP codecs in WiMAX under different network parameters and setting.

\section{A. Scenario 1}

In the first case, we measure the performance of VoIP codecs against the various performance matrices by considering the variable sizes of the RTP jitter buffer. We used 30ms packet stay time in jitter buffer, and find out how it affected the voice quality.

Fig. 2(a) and 2(b) show the average one-way delay and jitter with RTP buffer and $30 \mathrm{~ms}$ packet stay time. The results show that, under a heavy load, the G711 did not perform well and has highest delays $380 \mathrm{~ms}$ compared to other codecs. When the network becomes congested G711 reach to unsatisfied state while others codecs have normal behavior for 
each flow. In Fig. 2(b) G711 interestingly has minimum average jitter for two flows; no doubt it also has highest average jitter for some flows. Some time this type of situation is caused by wireless link quality. The average jitter for rest of codecs is almost same while G726 with $32 \mathrm{kbps}$ and $24 \mathrm{kbps}$ has ideal jitter under the simulated network setup.

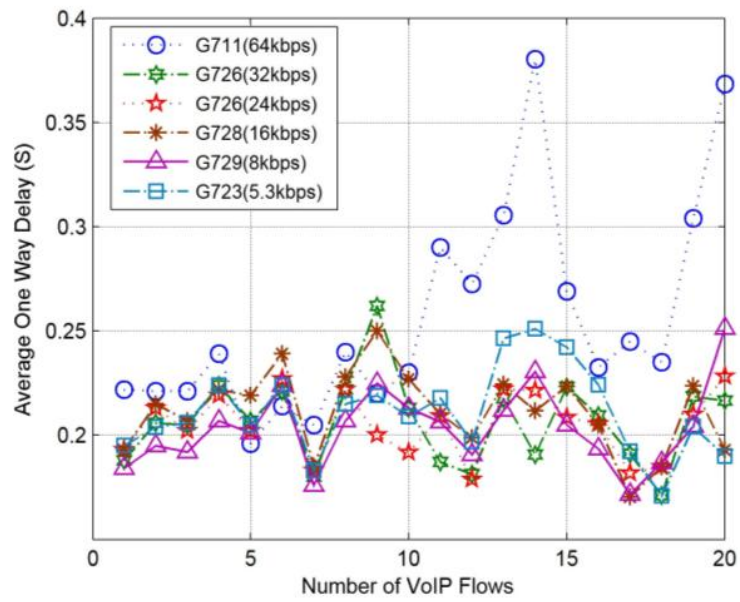

Fig. 2(a). Average One Way of various codecs with RTP jitter buffer.

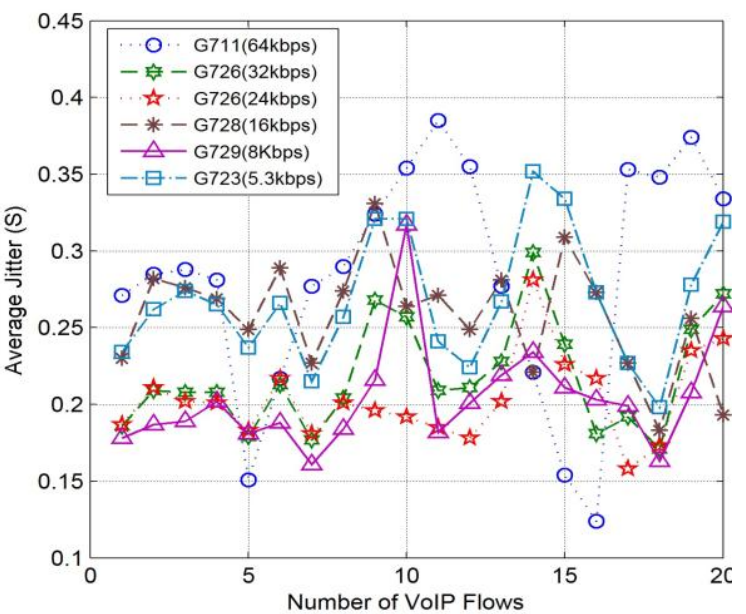

Fig. 2(b). Average jitter of various codecs with RTP jitter buffer.

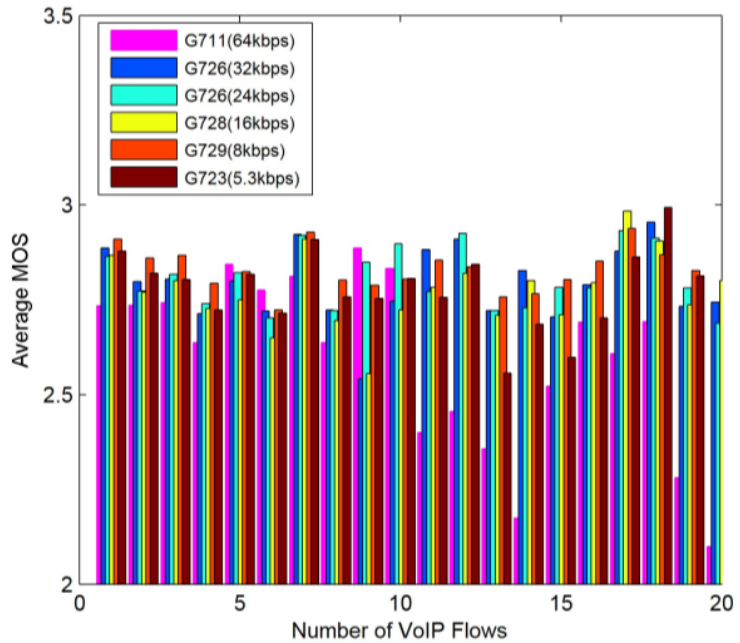

Fig. 3(a). Average Mean Opinion Score of various codecs with the RTP jitter buffer.

Fig. 3(a) shows the average MOS versus the selected codecs. G711 had acceptable MOS when the number of users was less and start to degrade as soon as the VoIP flows increased. Other Codecs almost have average MOS of 2.8 but among them G726 and G723 have smooth score during the whole period.

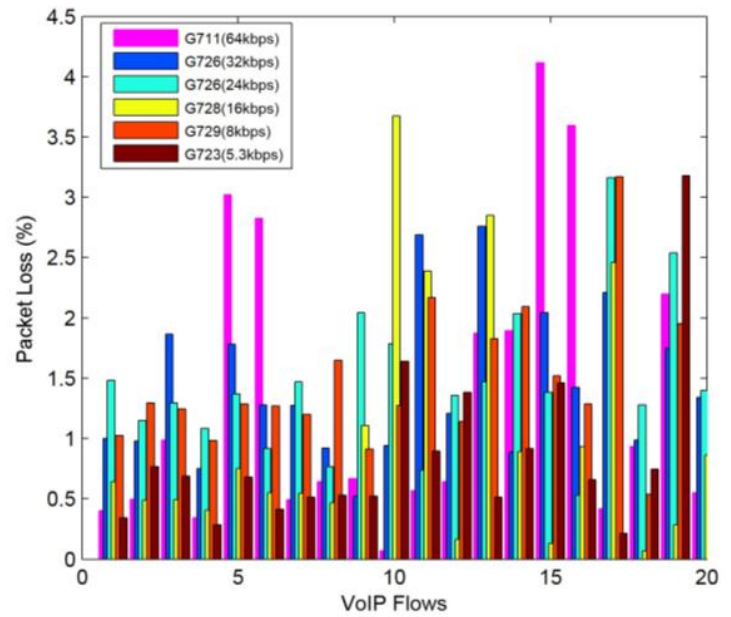

Fig. 3(b). Packet Loss of various codecs with the RTP jitter buffer

\section{B. Scenario 2:}

In this scenario, we evaluated the performance of VoIP flows without RTP jitter buffer over the BE scheduling class. Normally, the RTP jitter buffer is used to control unordered packets to improve the voice quality.

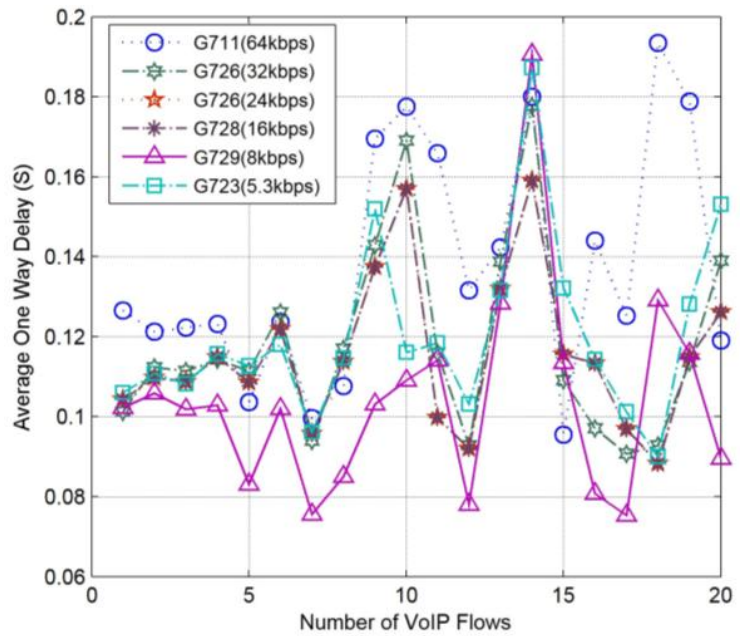

Fig. 5(a). Average One Way Delay of the various codecs without the RTP jitter buffer.

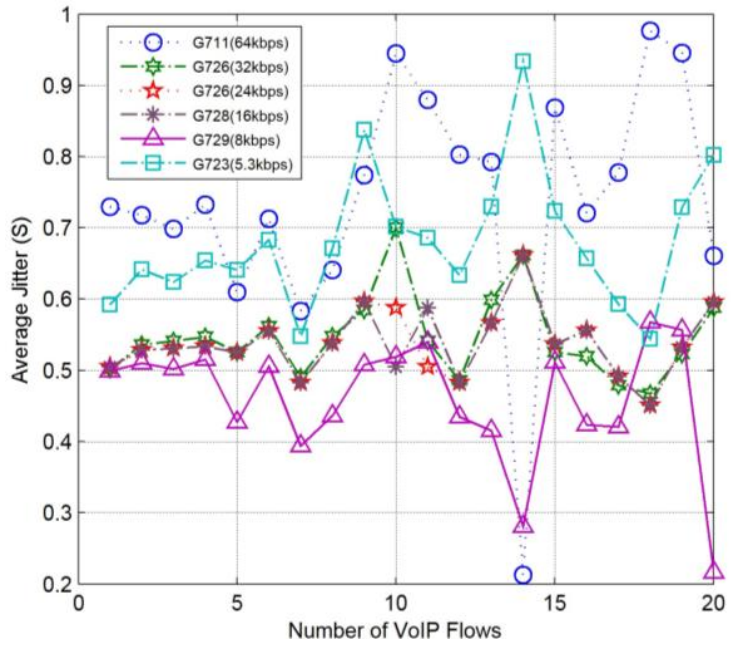

Fig. 5(b). Average jitter of the various codecs without the RTP jitter buffer. 
Fig. 5(a) shows fist five flows have symmetric delay, who arrived later those which have an asymmetric or unpredictable delay. We noticed G729 performed better than others codecs in this scenario as well as in first scenario too.. Interestingly, almost all of the used codecs have an average delay of $120 \mathrm{~ms}$. Fig. 5(b), indicates that G711 has higher jitter as compared to others. In contrast, G729 has lower jitter than others. The rest of the codecs have a different jitter value; around $600 \mathrm{~ms}$, which is undesirable for VoIP quality.

Fig. 6(a) shows the average MOS for different codecs. Approximately all flows have the same 3.1 Mean Opinion Score to measure the user perceived voice quality, at some extent which is satisfactory. On the other hand, one G729 flow has a minimum of $2.4 \mathrm{MOS}$; this may be due to the SS location or to weak signal strength. MOS for G711 also varies as usual after the 10th flow, when the network becomes overloaded with the arrival of a different traffic.

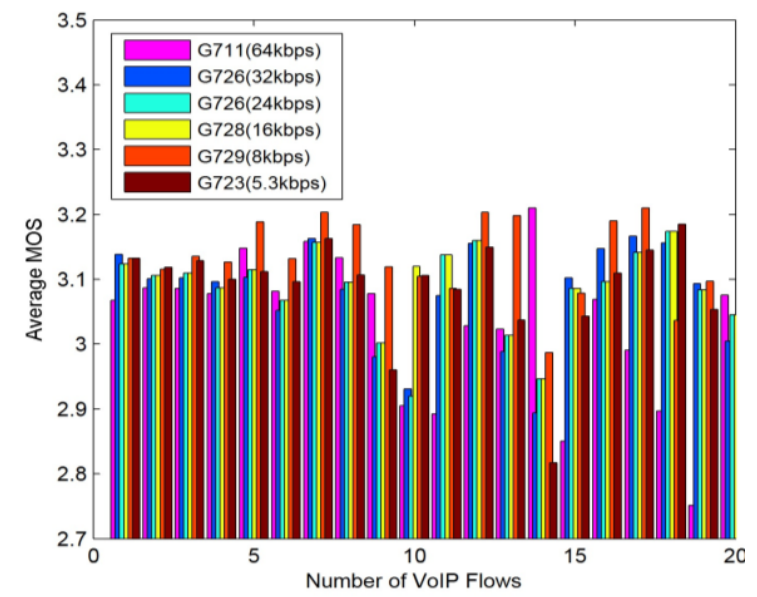

Fig. 6(a). Average MOS of various codecs without the RTP jitter buffer.

Fig. 6(b) shows the packet loss ratio for each codec against the number of VoIP flows. Only some flows have more than $2 \%$ packet loss while remaining flows have average packet loss less than $1 \%$. In the light of the above discussion we conclude that the referred VoIP service better performs without the RTP jitter buffer, because the packet received with less delay alternatively has higher jitter buffer. In this scenario, MOS is desirable, and also at a satisfactory level.

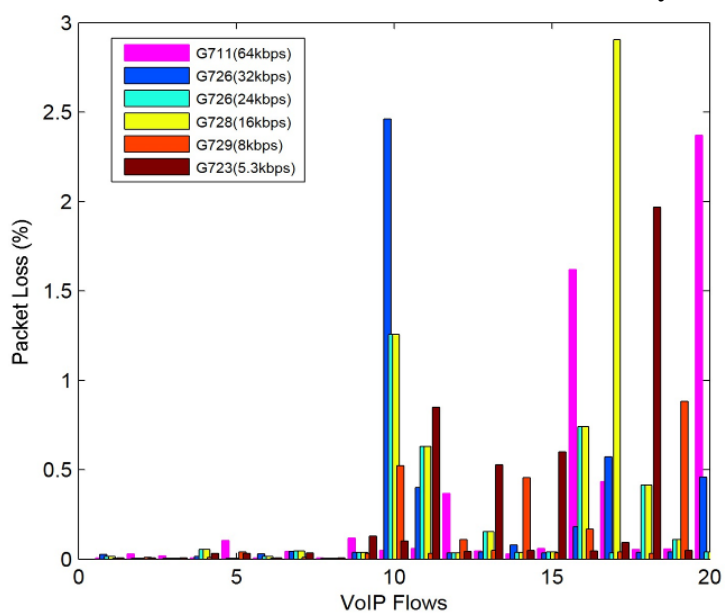

Fig. 6(b). Packet Loss of various codecs without RTP jitter buffer.

\section{CONCLUSION AND FUTURE WORK}

We evaluated the performance of VoIP codecs over the BE
WiMAX network by performing the simulation experiments in QualNet Simulation tool. We measured the VoIP performance over the Best Effort scheduling class with two experiments, one with the RTP jitter buffer and other one without jitter buffer. We found that the VoIP flows have better voice quality with higher MOS under no RTP jitter buffer as compared with the RTP jitter Buffer. Packet loss ratio is also noted twice time lower instead with jitter buffer. We also evaluated the various values for the packet stay time in jitter buffer and best one was used in our experiments. From this analysis, we conclude that the RTP jitter buffer has a significant effect on the overall performance of the VoIP application especially in the best effort scheduling class due to its lowest priority.

In future research, we have a plan to analyze the performance of a given VoIP application scenarios in a mobile environment with respect to the impact of the different mobility models. As suggested in [10], we also have a plan to evaluate and develop an efficient scheduler for given approach to sustain the minimum quality level.

\section{REFERENCES}

[1] N. Scalabrino, F. D. Pelegrini, I. Chlamtac, A. Ghittino, and S. Pera, "Performance evaluation of a WiMAX testbed under VoIP traffic," in Proc. First ACM International Workshop on Wireless Network Testbeds, Experimental evaluation and Characterization (WiNTECH), September 2006, PP. 97-98.

[2] IEEE 802.16 Working Group, IEEE Standard for Local and Metropolitan Area Networks, "Part 16: Air Interface for Fixed Broadband Wireless Access Systems", IEEE Std. 802.16-2004, October 2004.

[3] IEEE 802.16 Working Group, Amendment to IEEE Standard for Local and Metropolitan Area Networks, "Part 16: Air Interface for Fixed Broadband Wireless Access Systems - Physical and Medium Access Control Layer for Combined Fixed and Mobile Operation in Licensed Bands", IEEE Std. 802.16e- 2005, December 2005.

[4] K. Shuaib, "A Performance Evaluation Study of WiMAX Using QualNet," WCE, ICWN 2009, July 1-3, London, UK.

[5] R. M. Matos, P. Neves, and S. Sargento, "Evaluating WiMAX QoS performance in a real testbed," in Proc Conf. on Telecommunications ConfTele, Santa Maria da Feira, Portugal, May, 2009.

[6] F. Idrees and U. A. Khan "A Generic Technique for Voice over Internet Protocol (VoIP) Traffic Detection," IJCSNS, vol. 8, no. 2, February 2008.

[7] I. Adhicandra "Measuring Data and VoIP Traffic in WiMAX Networks," Journal of Telecommunication, vol. 2, issue 1, April 2010.

[8] Scalable-Network. [Online]. Available: shttp://Www.Scalable-Networks.Com.

[9] Scenarios. [Online]. Available: http://code.google.com/apis/talk/libjingle/scenarios.html

[10] R. Mahmood, M. I. Tariq et al., "A Novel Parameterized QoS based Uplink and Downlink Scheduler for Bandwidth/Data Management over IEEE 802. 16d Network," IJRTE, vol. 2, no. 1, November 2009, pp. $42-46$.

M. Imran Tariq received his MS degree in Computer Science from International Islamic University, Islamabad Pakistan, in 2008. He is currently pursuing his Ph.D. in Wireless Network from Japan Advanced Institute of Science and Technology, Japan. His research area includes wireless network, Peer-to-Peer network and VoIP over wireless network.

M. Ajmal Azad completed his BS degree in Computer sciences and MS in Electronics Engineering at International Islamic University, Islamabad Pakistan in 2004 and 2007 respectively. He is currently associated with Faculty of Engineering, University of Porto Portugal as a researcher and also 
pursuing Ph.D in Electrical and Computer Engineering from the same university. He was network manager at The Right Connection Pakistan, Multinet Private Limited Project. His research topic consists of Management and operations of next generation network (IMS, NGN, and VoIP), wireless network and P2P network.

Razvan Beuran received the B.Sc., M.Sc. and Ph.D. degrees from "Politehnica" University, Bucharest, Romania in 1999, 2000 and 2004, respectively (the $\mathrm{PhD}$ degree was delivered jointly with "Jean Monnet" University, Saint Etienne, France). From 2001 to 2005 he was with CERN, Geneva, Switzerland as a researcher. Since 2006 he is researcher with the National Institute of Information and Communications Technology,
Hokuriku StarBED Technology Center, Ishikawa, Japan. Since 2007 he is also project researcher with the Japan Advanced Institute of Science and Technology, Ishikawa, Japan. His research topics include network dependability studies in wired and wireless networks, in particular through the use of network emulation. He is a member of IEEE.

Yoichi Shinoda received his B.E., M.E. and Ph.D. from Tokyo Institute of Technology in 1983, 1985 and 1989, respectively. He joined Japan Advanced Institute of Science and Technology in 1991 as a professor of the School of Information Science. His research interests include distributed and parallel computing, networking systems, operating systems, and information environments. 